\title{
Erratum: Pill and Hyndman (2018)
}

In the article Pill, S., \& Hyndman, B. (2018). Gestalt psychological principles in developing meaningful understanding of games and sport in physical education. Journal of Teaching in Physical
Education, 37(4), 322-329, https://doi.org/10.1123/jtpe.2018-0033, incorrect images were used for Figures 1 and 2 . The online version of this article has been corrected. We apologize for this error. 